\title{
Selected clinical challenges of a supraclavicular cephalic vein in cardiac implantable electronic device implantation
}

\author{
E.B. Świętoń, R. Steckiewicz, M. Grabowski, P. Stolarz \\ Department of Cardiology, Medical University of Warsaw, Poland \\ [Received: 28 September 2015; Accepted: 10 November 2015]
}

Background: Supraclavicular variations of the cephalic vein (CV) are detected sporadically. A somewhat more common finding is a CV variation with the typical course of the main vessel but with an additional supraclavicular branch, called the jugulocephalic vein (JCV). The aim of the study was to detect supraclavicular CVs or $J C V s$ via intra-operative venography as well as assess their effects on primary and later revision cardiac implantable electronic device (CIED) procedures in our patients. Materials and methods: We analysed venographic images obtained during CIED procedures at our centre between 2011 and 2015. Out of the 324 venographies conducted during first-time CIED implantation, we identified 14 showing either a supraclavicular course of the CV itself or a persistent JCV. Among revision procedure venographies, we identified 1 case of pertinent $C V$ variations. These vessels had been morphometrically altered by previous medical interventions.

Results: Based on topography and morphometric parameters, we identified three anatomical variations of supraclavicular vessels: 2 cases of a supraclavicular CV and 12 cases of an infraclavicular CV accompanied by a persistent supraclavicular $J C V$ (with the diameter larger than that of the main CV in 5 cases and smaller in 7 cases). In 2 cases the enlarged diameter of the JCV was probably due to increased collateral venous flow resulting from thrombotic lesions in the subclavian vein. Conclusions: Supraclavicular CV variations are rare. Nonetheless, they may significantly affect both first-time and later revision CIED procedures. The presence of a supraclavicular vein is an indication for diagnostic venography in the area of the clavipectoral triangle before the CIED procedure. (Folia Morphol 2016; 75, 3: 376-381)

Key words: venography, cephalic vein, jugulocephalic veins, cardiac implantable electronic device

\section{INTRODUCTION}

The mainstream approach to cardiovascular lead insertion during cardiac implantable electronic device (CIED) placement procedures is transvenous [4]. Both the topography and morphometric parameters of the cephalic vein (CV) along its deltopectoral grove segment are generally favourable for the use of this vessel in CIED procedures. In fact, the use of CV cut-down approach as the preferable cardiac lead insertion point is characterised by a lower risk of complications than the axillary vein or subclavian vein (AV/SV) puncture $[3,7,13]$.

The CV collects blood from superficial veins of the hand and subsequently drains into the AV or, less commonly, the SV in the clavipectoral triangle. This is where the CV is found in $95 \%$ of autopsies [5].

Address for correspondence: R. Steckiewicz, MD, PhD, Department of Cardiology, Medical University of Warsaw, ul. Banacha 1A, 02-097 Warszawa, Poland, tel: +48 22599 2958, e-mail: r.steckiewicz@pro.onet.pl 


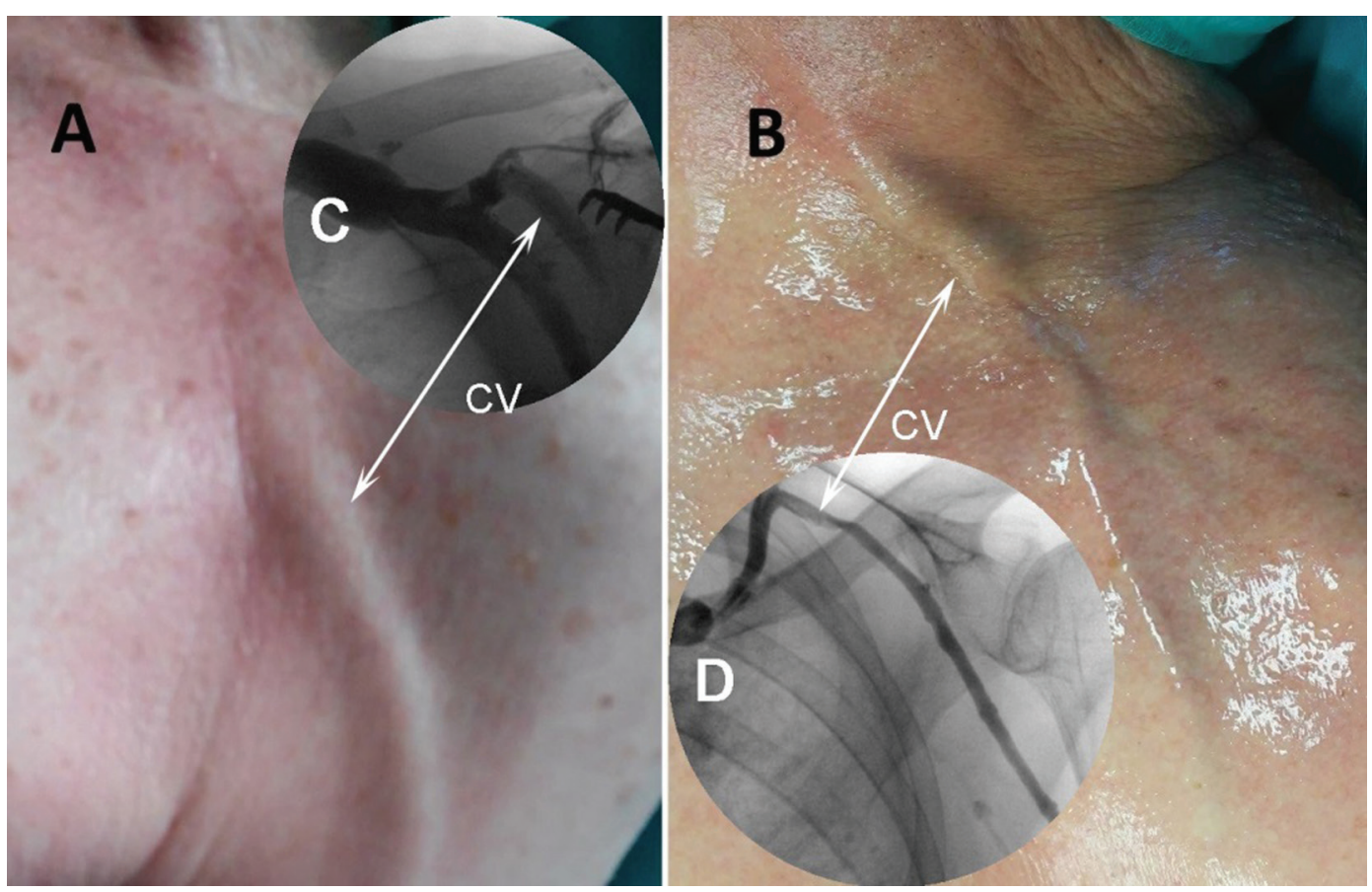

Figure 1. The infraclavicular and supraclavicular position of the cephalic vein (CV) apparent in the clavipectoral triangle on physical examination and on contrast-enhanced fluoroscopy (white arrows); A, C. Typical anatomical layout with the site of CV-subclavian vein confluence located below the clavicle ("CV disappears below the clavicle"); B, D. A CV variation comprising a single vessel coursing over the clavicle (supraclavicular) prior to its confluence with the external jugular vein.

Not unlike other veins, the course of the CV is characterised by a greater inter-individual variability than that of arteries, with the typical CV course involving its drainage into the AV/SV beneath the clavicle (Fig. 1A). A supraclavicular course of the CV is found sporadically during CIED procedures and has been reported in $0.2 \%$ of relevant autopsy examinations. Another, slightly more common, CV variation has the main vessel in its typical infraclavicular position but features an accessory supraclavicular branch corresponding to a persistent jugulocephalic vein (JCV) $[1,4,8,9,14]$.

Both anatomical variations of the CV may affect the initial stage of CIED implantation procedures as well as have long-term clinical sequelae. This paper presents more extensive clinical material with more numerous pictures in comparison to individual, supraclavicular CV case reports typically found in the literature.

\section{MATERIALS AND METHODS}

Our data were collected over 4 years of CIED procedures conducted at our centre (January 2011- August 2015). We analysed fluoroscopy venograms recorded as part of two types of procedures: firsttime CIED implantation and CIED re-implantation procedures, the latter due to lead dysfunction and/ /or device upgrade.
Venograms were obtained in the case of difficulties with lead insertion or advancement within the $\mathrm{CV}, \mathrm{AV}$ or SV and whenever precise AV/SV imaging was required due to unsuccessful attempts at AV/SV puncture.

Out of 2,036 first-time CIED recipients, 324 patients ( 130 women and 194 men; mean age $73 \pm 10$ ) underwent intraoperative venography procedures. In 14 cases venograms showed the presence of a supraclavicular vessel, either in the form of a solitary supraclavicular CV (2 cases) or a supraclavicular persistent JCV (12 cases) draining into the main CV. In the latter configuration, the 2 vessels were found to have various diameter ratios. In 2 cases of a JCV with a diameter larger than that of the 'main' vessel, this $\mathrm{CV}$ variation was accompanied by clinically silent venous thrombosis causing complete or significant SV occlusion, which most likely contributed to the change in the original diameter of these vessels.

Out of 213 CIED revision procedures, there was 1 case of lead insertion via a supraclavicular variant of the CV.

Venograms were obtained with the OEC 9900 Elite workstation (GE) in the antero-posterior view. We evaluated dynamic contrast flow (cine-mode) and static fluoroscopy images of the contrast-enhanced $\mathrm{CV}$ in the area of the clavipectoral triangle from the 

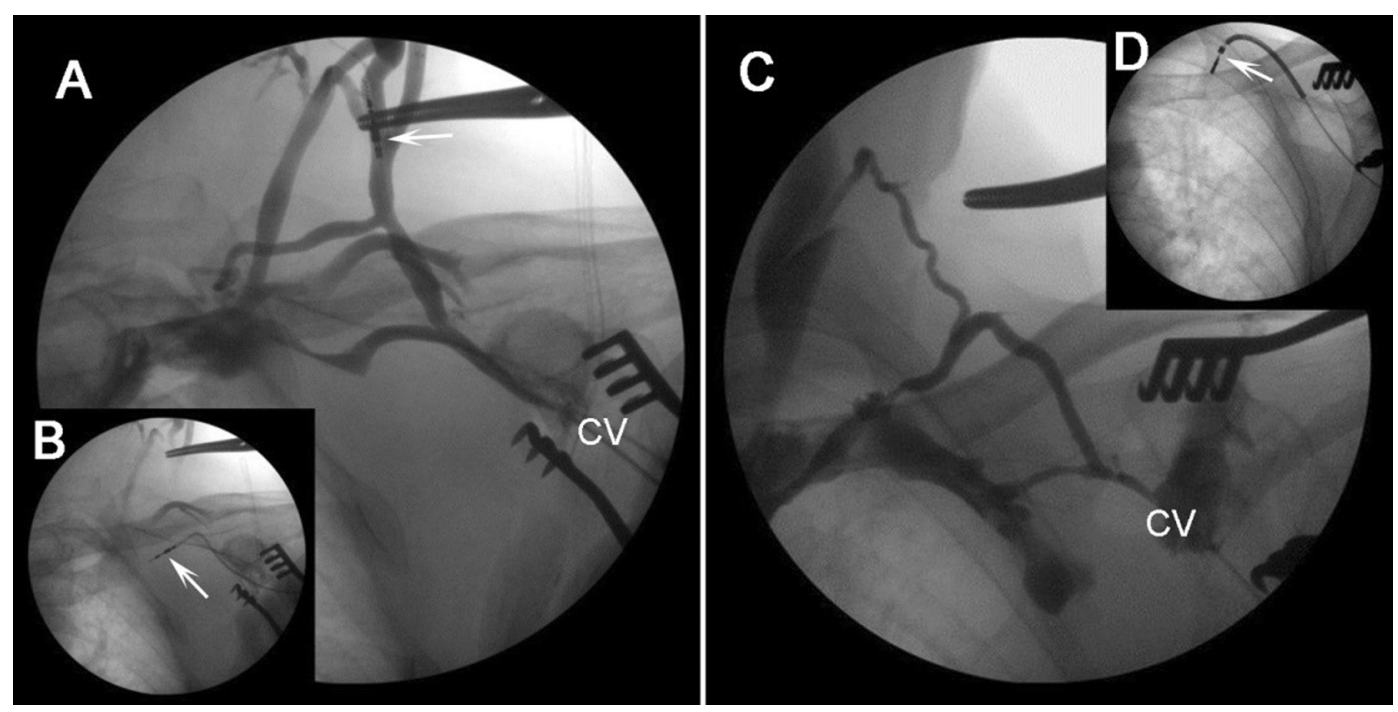

Figure 2. Anatomical cephalic vein (CV) variations involving a supraclavicular jugulocephalic vein (JCV) with a diameter larger than that of the 'main' vessel (selective venography images); A. Morphometric parameters of the supraclavicular JCV precluded successful lead advancement (white arrow); B. Insertion of a cardiac stimulation lead via the 'main' vessel was obstructed by a stenosed CV-subclavian vein confluence (white arrow); C, D. Insertion of a cardiac defibrillation lead was obstructed by a narrowing at the site where the supraclavicular JCV drained into the 'main' CV (white arrow).
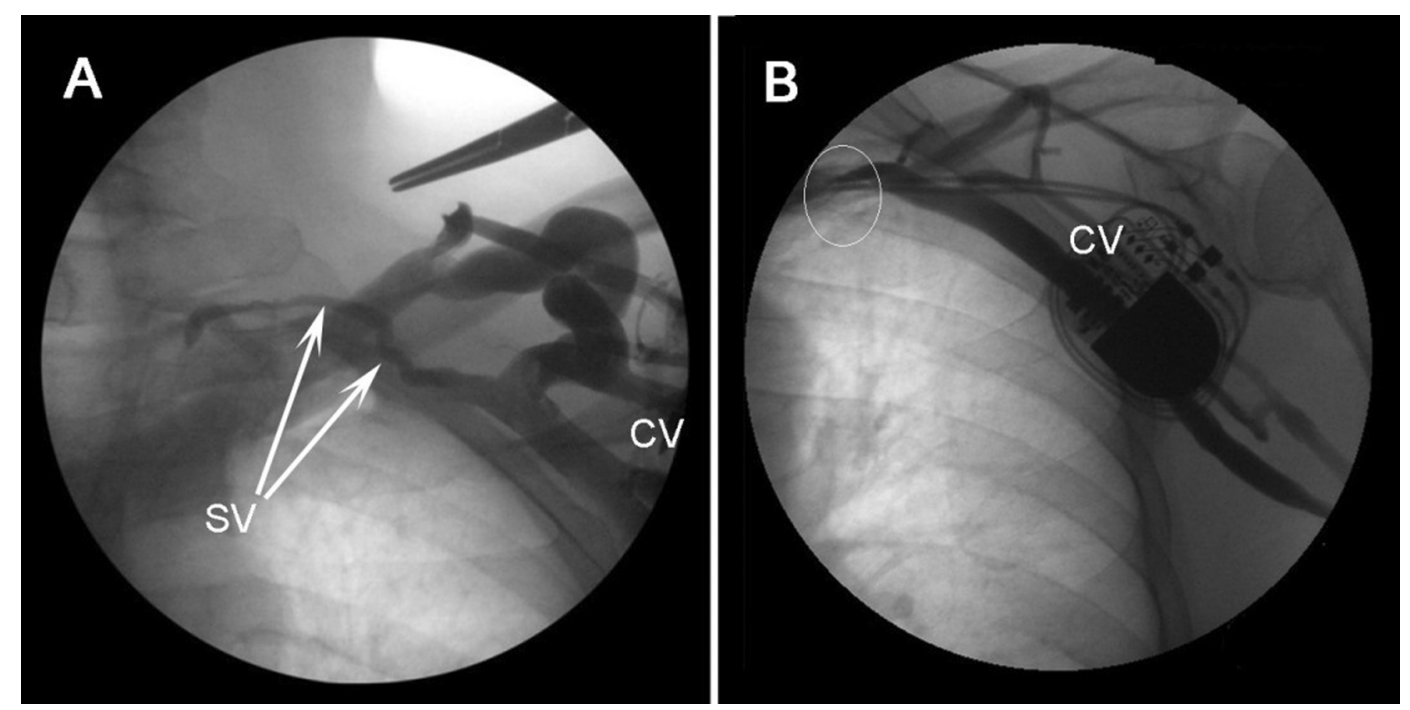

Figure 3. Clinically silent venous thrombosis in the lumen of the left subclavian vein (SV), with collateral circulation via a large-diameter supraclavicular jugulocephalic vein (JCV) constituting a natural venous by-pass. Clear contrast retention in peripheral segments of affluent vessels (conventional venography); A. A first-time cardiac implantable electronic device (CIED) procedure: significant stenosis of the SV lumen and a large-diameter supraclavicular JCV; B. Revision CIED procedure involving an attempt to introduce a new ventricular lead as the original lead, implanted together with a DDD system many years earlier, had been damaged; visible SV lumen occlusion along the course of the leads (white oval); CV — cephalic vein.

level of CV dissection to the level of CV confluence with the AV/SV.

The selected, pertinent venographic images can be divided into two types: conventional venographies, with the contrast agent administered into distal veins of the hand/forearm, and selective CV procedures, with contrast administration into the proximal segment of the CV within the surgical field. Images of the latter type are characterised by virtually or completely invisible collateral vasculature distal to the site of contrast administration.

\section{RESULTS}

The 14 identified cases of CV variation out of 324 venographies conducted during first-time CIED implantation procedures were divided into three 

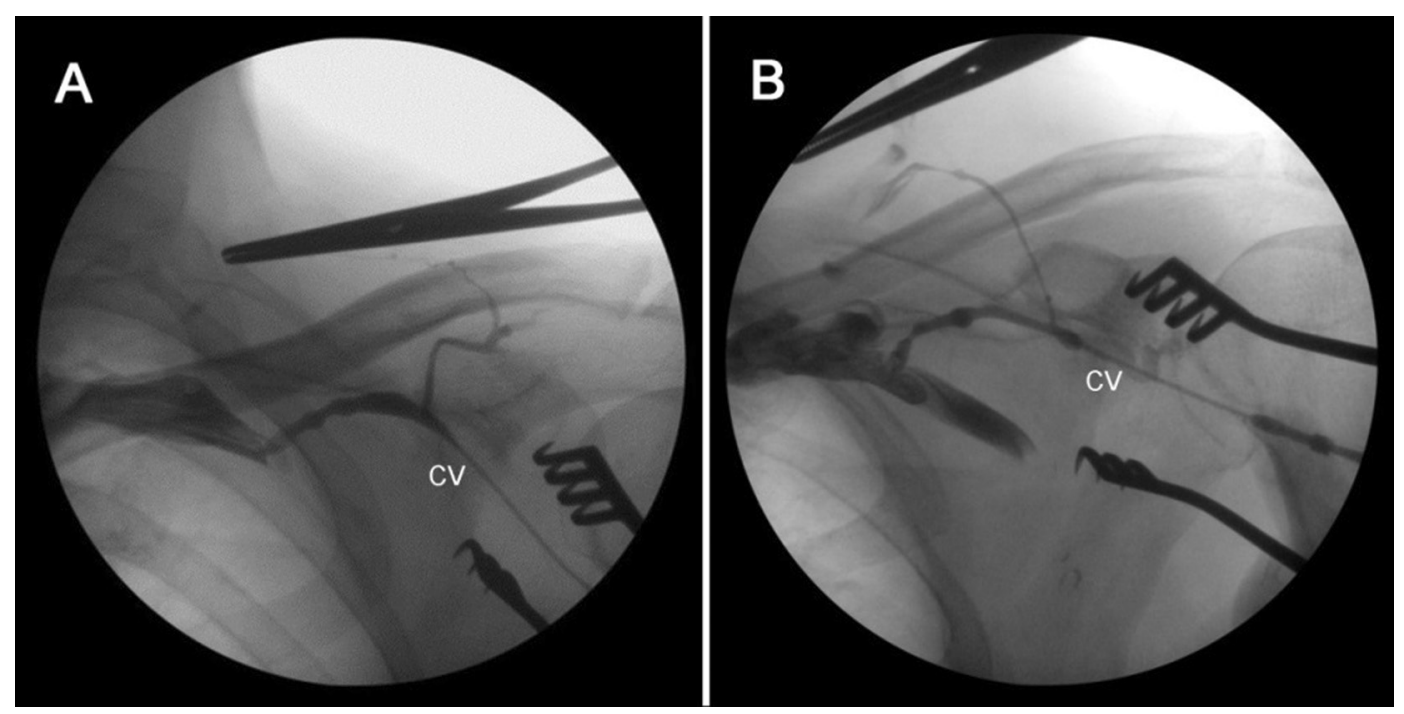

Figure 4. A, B. Images of a supraclavicular jugulocephalic vein (JCV) whose diameter was smaller than that of the main vessel. The branching site of these vessels in the surgical field in the deltopectoral groove (selective venography). CV — cephalic vein.
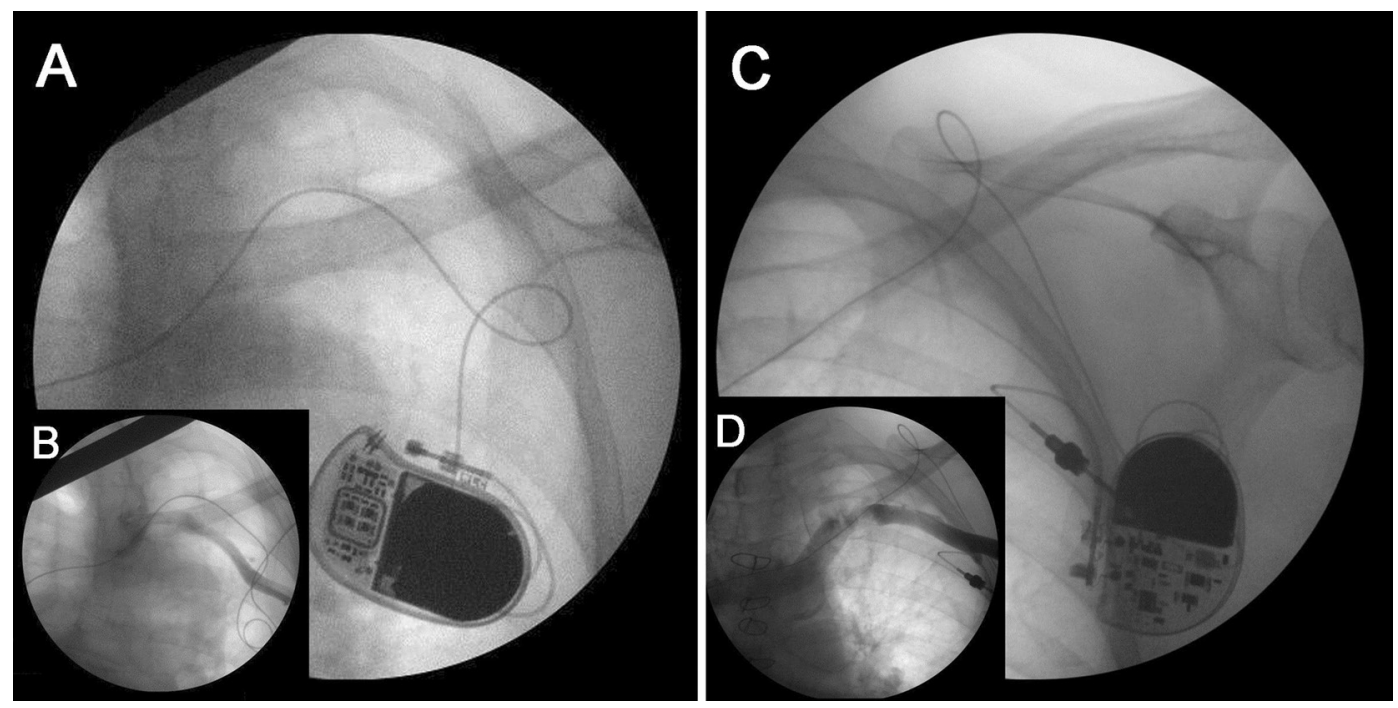

Figure 5. A. A comparison of 2 cases with cardiac leads advanced via supraclavicular vessels. Venographic images were obtained during elective replacement indicator (ERI)-related device replacement procedures associated with new lead placement; unobstructed contrast flow via the patent axillary vein an subclavian vein; no contrast enhancement of the cephalic vein (CV); B. Status post implantation of a VVI system with a unipolar lead inserted into the venous system through a supraclavicular jugulocephalic vein years earlier; C, D. Status post implantation of a VVI system with a lead inserted via external jugular vein cut-down — due to CV absence.

distinct anatomical categories for the purpose of this study. There were no identifiable clinical causes behind the configuration and morphometric structure of the identified $14 \mathrm{CV}$ variations.

One CV variation - observed in 2 cases - was a single $\mathrm{CV}$ coursing supraclavicularly prior to its confluence with the external jugular vein (Fig. 1B).

Another CV variation - found in 5 cases - was an accessory vessel, consistent with a persistent JCV, coursing above the clavicle and draining into the main $\mathrm{CV}$ within the bounds of the clavipectoral triangle. In these cases, the diameter of the persistent JCV tended to be larger than that of the typically positioned main CV (Fig. 2A-D).

Out of these 5 cases, a persistent JCV of a diameter larger than that of the 'main' vessel was in 2 cases accompanied by clinically silent venous thrombosis causing complete or significant SV occlusion (Fig. 3A, B).

The third $C V$ variation was also the most common one (found in 7 cases) and involved a supraclavicular persistent JCV whose diameter was smaller than that of the main vessel (Fig. 4A, B). 
Among the 213 revision procedures conducted during the analysed period there was 1 case where a unipolar lead had been advanced through a supraclavicular CV during a VVI system implantation many (> 25) years earlier (Fig. 5A, B).

\section{DISCUSSION}

The CV has been used during CIED implantation procedures to insert cardiac leads via a venous cutdown technique [2]. The $\mathrm{CV}$ is one of superficial veins of the upper extremity and typically drains medially into the AV below the clavicle, after piercing the clavipectoral fascia (Fig. 1A) [8].

The presence and nature of any individual variations in the morphological structure and anatomical position of vessels can be detected practically only during intraprocedural venography, in the cases of lead advancement difficulties [11, 12]. The purpose of this study was to use venographic images to assess the rate and nature of two CV variations (a supraclavicular CV and a supraclavicular persistent JCV) as well as their effect on the course of CIED implantation procedures.

A supraclavicular $\mathrm{CV}$ is a rarely found and rarely reported anatomical variation, which is consistent with our findings (Fig. 1B) $[1,4,6,10,14]$. If detected during a CIED procedure, a supraclavicular CV typically affects lead insertion. Before the AV/SV puncture technique, this CV variation typically necessitated ipsilateral jugular vein cut-down or the use of the contralateral CV (Fig. 5C, D).

This study demonstrated that the CV variation found equally rarely was a supraclavicular persistent $\mathrm{JCV}$ draining into the main $\mathrm{CV}$ and of a diameter larger than that of the main vessel (Fig. 2A-D).

Before cardiac lead insertion kits became available, successful lead advancement in such and similar cases could sometimes be obtained by the use of unipolar leads (thanks to their smaller diameter and favourable lead tip structure) (Fig. 5A, B). The CV variations mentioned above allowed for pacemaker implantation without the necessity of jugular vein dissection and their many-year follow-up demonstrated successful lead placement without local adverse effects.

Physical properties and structure of modern bipolar leads make it more challenging to advance them via such supraclavicular vessels during implantation of a cardiac pacemaker and even more so in the case of implantable cardioverter-defibrillator implantation (Fig. 2C, D).
The most commonly found CV variation was a normally positioned main vessel draining a supraclavicular persistent JCV of a considerably smaller diameter (Fig. 4A, B). Although the presence of such a branch did not directly affect the course of the procedure, we believe that in some cases it might be of significance after the procedure.

The anatomical position of the SV between two bones from the lateral margin of the first rib to the level of sternal end of the clavicle facilitates the development of venous thrombosis, which may significantly obstruct blood flow while remaining clinically silent. In such cases the presence of a supraclavicular JCV may help in the development of collateral circulation involving enlargement of its lumen due to the compensatory increase in blood flow (Fig. 3A).

We are aware of the considerable limitations of our study, e.g. venographic assessments of vessels in the clavipectoral triangle was performed only on the side of the procedure and only in cases of potential or already encountered difficulties. The actual prevalence of the described $\mathrm{CV}$ variations seems to be higher; however, not every patient had had indications for venography. Moreover, our theory on the CV branch lumen widening as a result of compensatory blood flow in the case of SV occlusion was not accompanied by imaging documentation of the initial status of the vessel, etc.

We believe this study to be one of the few presenting the practical aspect of rare, and thus rarely reported, CV variations in a larger group of patients.

\section{CONCLUSIONS}

The presented anatomical variations of the $\mathrm{CV}$ are rare; however, they can significantly affect the course of primary and revision CIED implantation procedures.

The presence of a supraclavicular vein should be an indication for diagnostic venography of the vessels to be used during the CIED implantation procedure.

\section{REFERENCES}

1. Kim D-I, Han S-H (2010) Venous variations in neck region: cephalic vein. IJAV, 3: 208-210.

2. Knight BP, Curlett K, Oral H, Pelosi F, Morady F, Strickberger SA (2002) Clinical predictors of successful cephalic vein access for implantation of endocardial leads. J Interv Card Electrophysiol, 7: 177-180.

3. Kolettis T M, Lysitsas D N, Apostolidis D, Baltogiannis G G, Sourla E, Michalis L K (2010) Improved 'cut-down' technique for transvenous pacemaker lead implantation. Europace, 12: 1282-1285. doi: org/10.1093/europace/ euq173 
4. Lau EW (2007) Upper body venous access for transvenous lead placement-review of existent techniques. Pacing Clin Electrophysiol, 30: 901-909. doi: 10.1111/j.15408159.2007.00779.x.

5. Loukas M, Myers CS, Wartmann ChT, Tubbs RS, Judge T, Curry B, Jordan R (2008) The clinical anatomy of the cephalic vein in the deltopectoral triangle. Folia Morphol, 67: 72-77.

6. Lum C, Ladenheim ED (2013) An interesting clinical case: variant of the cephalic vein emptying into the internal jugular vein. Semin Dial, 26: E11-E12. doi: 10.1111/j.1525139X.2012.01102.x.

7. Parsonnet V, Roelke M (1999) The cephalic vein cutdown versus subclavian puncture for pacemaker/ICD lead implantation. PACE, 22: 695-697.

8. Plakornkul V, Manoonpol Ch (2006) The patterns of the cephalic veins termination. Siriraj Med J, 58: 1204-1207.

9. Saaid A, Drysdale I (2008) Unusual termination of the cephalic vein. Clin Anat, 21: 786-787. doi: 10.1002/ ca.20661.

10. Świętoń E, Steckiewicz R, Stolarz P, Górko D, Grabowski M (2015) Supraclavicular course of the cephalic vein - implications for cardiac electronic device implantation. Folia Cardiol, 10: 200-203. doi: 10.5603/FC.2015.0034.

11. Tokano T, Nakazato Y, Shiozawa T, Konishi H, Hiki M, Kato Y, Komatsu S, Yamase M, Komatsu K, Hayashi H, Sekita G, Suwa S, Bito F, Kizu K, Sumiyoshi M, Daida H (2013) Variations in cephalic vein venography for device implantation-Relationship to success rate of lead implantation. J Arrhyth, 29: 9-12. doi: org/10.1016/j.joa.2012.05.009.

12. Tse HF, Lau CP, Leung SK (2001) A cephalic vein cutdown and venography technique to facilitate pacemaker and defibrillator lead implantation. Pacing Clin Electrophysiol, 24: 469-473.

13. Ussen B, Dhillon PS, Anderson L, Beeton I, Hickman M, Gallagher MM (2011) Safety and feasibility of cephalic venous access for cardiac resynchronization device implantation. Pacing Clin Electrophysiol, 34: 365-369. doi: 10.1111/j.1540-8159.2010.02975.x.

14. Wysiadecki G, Polguj M, Topol M (2016) Persistent jugulocephalic vein: case report including commentaries on distribution of valves, blood flow direction and embryology. Folia Morphol, 75: 271-274. doi: 10.5603/ FM.a2015.0084. 